\title{
Editorial
}

\section{Differential Equation Models in Applied Mathematics: Theoretical and Numerical Challenges}

\author{
Fasma Diele (1)
}

Citation: Diele, F. Differential

Equation Models in Applied

Mathematics. Mathematics 2022, 10,

249. https://doi.org/10.3390/

math10020249

Received: 4 January 2022

Accepted: 11 January 2022

Published: 14 January 2022

Publisher's Note: MDPI stays neutral with regard to jurisdictional claims in published maps and institutional affiliations.

Copyright: (c) 2022 by the author. Licensee MDPI, Basel, Switzerland. This article is an open access article distributed under the terms and conditions of the Creative Commons Attribution (CC BY) license (https:// creativecommons.org/licenses/by/ $4.0 /)$.
Istituto per Applicazioni del Calcolo ‘M.Picone', CNR, Via Amendola 122/D, 70126 Bari, Italy; fasma.diele@cnr.it

\section{Motivations for the Special Issue}

The articles published in the Special Issue "Differential Equation Models in Applied Mathematics: Theoretical and Numerical Challenges" of the MDPI Mathematics journal are here collected. The Special Issue intended to highlight old and new challenges in the formulation, solution, understanding, and interpretation of models of differential equations (DEs) in different real world applications. Indeed, models of differential equations can describe complex mechanisms arising in a wide range of applications in many different sectors as ecology, health, biology, economics, and finance. Differential modelling and difference equations are tools to understand the dynamics, do forecasting and scenario analysis; in addition, they allow for the detection of optimal solutions according to selected criteria.

The technical topics covered in the seven articles published in this book include: asymptotic properties of high order nonlinear DEs [1,2], analysis of backward bifurcation [3], stability analysis of fractional-order differential systems [4]. Models oriented to real applications consider the chemotactic between cell species [5], the mechanism of on-off intermittency in food chain models [6] and the occurrence of hysteresis in marketing [3]. Numerical aspects deal with the preservation of mass and positivity [5] and the efficient solution of Boundary Value Problems (BVPs) for optimal control problems [7].

In the following, I summarize the main content of novelty of this book distinguishing among contributes that concerns:

- $\quad$ Theoretical challenges of DEs [1,2,4];

- $\quad$ Numerical challenges of DEs [5,7];

- $\quad$ Real-word applications of DEs $[3,6]$.

\subsection{Theoretical Challenges of DEs}

In articles [1,2] the focus is on high-order differential equations. In [1], new oscillation theorems for fourth-order differential equations are established using the Riccati and the integral averaging techniques. The article [2] investigates the inverse problem for a non homogeneous, higher-order Sobolev type equation with assigned Cauchy and overdetermined conditions. By using the theory of bounded polynomial operator pencils, the problem is initially reduced into two regular and singular aggregates and then it is restored using the method of successive approximations. A theorem on the solvability of the original problem represents the main theoretical contribute of this paper to the literature on this subject.

In the review article [4] systems of fractional-order DEs with Caputo derivative are presented. Due to the dependence on the order of the fractional derivatives, the linear stability analysis leads to properties fundamentally different from those of classical DEs: unlike systems of integer order, coefficients of the systems are not sufficient to describe stability properties of solutions. By reviewing the asymptotic analysis of the Mittag-Leffler function and of its derivatives and by examining systems with some specific structures, this paper intends to contribute to the research on the stability analysis of multi-order higher dimensional systems that represents nowadays an important theoretical challenge for fractional-order DEs. 


\subsection{Numerical Challenges of DEs}

The transmission model for microfluidic chips presented in the featured paper [5] involves doubly parabolic DEs in 2D spatial domains connected with either a doubly parabolic or a hyperbolic-parabolic DEs in 1D domains. The important contribute of this paper is the development of novel positive numerical conditions and numerical methods, based on finite difference schemes, assuring the mass-preservation at the external boundaries and the interfaces between domains of different sizes. It has to be underlined that is the first numerical work where this new technique of switching the size of the domains and type of partial differential equations, i.e., parabolic vs. hyperbolic, is introduced in the literature.

In paper [7], Hamiltonian boundary valued DEs deriving from the applications of the indirect method, based on Pontryagin's conditions, to optimal control problems are considered. The main contribute of this paper is to show how to properly choose and use codes on popular scientific platforms (Fortran, Matlab, R), for solving some specific challenging optimal control problems. This paper gives important indications useful to choose an initial mesh, to handle the input parameters or to use of a continuation technique for nonlinear problems to achieve accurate solutions via a bvp (boundary value problem) solver.

\subsection{Real-Word Applications of DEs}

In the featured paper [6], the power of DEs as leading mathematical tools for describing ecosystem dynamics is illustrated. In particular, some preliminary steps towards a conceptual description of population outbursts grounded into an environment-driven mechanism are described. The focus is on a three-species food chain represented by the Hastings-Powell model: by stochastically perturbing the value of some parameters, the authors show the emergence of on-off intermittency, i.e., an irregular alternation between stable phases and sudden bursts in population size. The strength of this paper lies in representing the first evidence of the possibility of on-off intermittent behavior in a food chains model.

The original point of view adopted in the paper [3] illustrates the ability of DEs in modelling and anlyzing dynamical scenarios in different real-word applications. Here the expert approach of the author adopts the analogy between the key mechanism of contagion for both the spread of an epidemic and for a referral marketing defined as viral because of it involves a person-to-person transmission. The theoretical concept of backward bifurcation, to avoid in the epidemic context, in the viral marketing could strengthen the campaign's chances of survival. However, the paper points out the possible introduction of a risk factor in the bistability range where, according to the chosen initial conditions, hysteresis-type behaviors can emerge.

\section{Conclusions}

I hope that this collection will be useful for those working in the area of modelling real-word applications through differential equations and those who care about an accurate numerical approximation of their solutions. The reading is also addressed to ones who are willing to become familiar with differential equations which, due to their predictive abilities, represent the main mathematical tool for making scenario analysis of our changing world [8].

Funding: This research received no external funding

Acknowledgments: As the guest editor, I want to thank all the authors for contributing to the Special Issue with their interesting and valuable articles collected in this book. I would also to thank all referees for their thorough and timely reports on the submitted works. Finally, it is my pleasure also to thank, in person of Grace Du, all the editorial staff of the journal Mathematics for the pleasant cooperation, during the preparation of the Special Issue and during the preparation of this book.

Conflicts of Interest: The author declares no conflict of interest. 


\section{References}

1. AlGhamdi, A.; Bazighifan, O.; El-Nabulsi, R.A. Important criteria for asymptotic properties of nonlinear differential equations. Mathematics 2021, 9, 1659. [CrossRef]

2. Zamyshlyaeva, A.; Lut, A. Inverse Problem for the Sobolev Type Equation of Higher Order. Mathematics 2021, 9, 1647. [CrossRef]

3. Lacitignola, D. Handling Hysteresis in a Referral Marketing Campaign with Self-Information. Hints from Epidemics. Mathematics 2021, 9, 680. [CrossRef]

4. Brandibur, O.; Garrappa, R.; Kaslik, E. Stability of Systems of Fractional-Order Differential Equations with Caputo Derivatives. Mathematics 2021, 9, 914. [CrossRef]

5. Braun, E.C.; Bretti, G.; Natalini, R. Mass-preserving approximation of a chemotaxis multi-domain transmission model for microfluidic chips. Mathematics 2021, 9, 688. [CrossRef]

6. Vissio, G.; Provenzale, A. On-Off Intermittency in a Three-Species Food Chain. Mathematics 2021, 9, 1641. [CrossRef]

7. Mazzia, F.; Settanni, G. BVPs Codes for Solving Optimal Control Problems. Mathematics 2021, 9, 2618. [CrossRef]

8. Cuddington, K.; Fortin, M.J.; Gerber, L.; Hastings, A.; Liebhold, A.; O'connor, M.; Ray, C. Process-based models are required to manage ecological systems in a changing world. Ecosphere 2013, 4, 1-12. [CrossRef] 